\title{
Online Wear Detection Using High-Speed Imaging
}

\author{
Seyfollah Soleimani, ${ }^{1, *}$ Jacob Sukumaran, ${ }^{3}$ Koen Douterloigne, ${ }^{2}$ Patrick De Baets, ${ }^{3}$ and \\ Wilfried Philips ${ }^{2,4}$ \\ ${ }^{1}$ Department of Computer Engineering, Faculty of Engineering, Arak University, Arak 38156-8-8349, Iran \\ ${ }^{2}$ Ghent University iMinds-Telin-IPI, St-Pietersnieuwstraat 41, B-9000 Gent, Belgium \\ ${ }^{3}$ Ghent University, Laboratory Soete, Technologiepark, Zwijnaarde 903, B-9052 Gent, Belgium \\ ${ }^{4}$ Senior member of IEEE
}

\begin{abstract}
In this paper, the change detection of a fast turning specimen is studied at micro-level, whereas the images are acquired without stopping the rotation. In the beginning of the experiment, the imaging system is focused on the surface of the specimen. By starting the rotation of the specimen, the diameter of the specimen changes due to wear, which results in de-focusing of the imaging system. So the amount of blur in the images can be used as evidence of the wear phenomenon. Due to the properties of the microscope, the corners of the frames were dark and had to be cropped. So, each micrograph reflects only a small area of the surface. Nevertheless, techniques like stitching of multiple images can provide a significant surface area for micro-level investigation which increases the effectiveness of analyzing the material modification. Based on the results computer vision could detect a change of about $1.2 \mu \mathrm{m}$ in the diameter of the specimen. More important is that we could follow the same locations of the surface in the microscopic images despite blurring, uneven illumination, change on the surface, and relatively a high-speed rotation.
\end{abstract}

Key words: Rolling-sliding, optical microscopy, micro-scale abrasion, surface analysis

\section{INTRODUCTION}

Contact between surfaces is a common phenomenon in industrial equipment and components. These contacting surfaces are employed in places where motion transfers from one to another component. In such a situation, wear of the contacting surfaces is inevitable due to particle generation by means of different mechanisms (abrasion, adhesion, and fatigue). The severity of the wear depends on different factors such as friction, operating condition, materials, and the interface condition. Increasing wear causes breakdown of equipment due to progressive wear. In some situations, the damage may be irreversible. Thus, the wear process should be understood and with this knowledge a wear resistance material may be designed. Although the problem of wear analysis has received intense attention, there is still room for improvement and there are many possibilities to be investigated.

Traditional methods for wear analysis use a gravimetric analysis, monitoring of the dimensional change, and profilometry (Bayer, 2004). However, wear is investigated from the contact surface using different techniques such as scanning electron microscopy (SEM), optical microscopy, scanning tunneling microscopy, atomic force microscopy, and transmission electron microscopy (Sukumaran, 2014). Besides surface scars visible in two dimensional and three dimensional images, the topographic characteristics also elucidate the surface state. The commonly used technique for topography characterization is stylus profilometry. In this traditional

Received December 27, 2015; accepted June 7, 2016

${ }^{*}$ Corresponding author. s-soleimani@araku.ac.ir technique, the stylus makes contact with the surface which may damage the surface and also limit the processing speed (Kumar et al., 2005). Another difficulty with using a contacting stylus is that usually only one or a few lines on the surface can be measured for analysis for practical reasons. Moreover, contacting styli cannot be used online. To tackle these problems, vision systems have been used, which eliminates contact with the surface, allows analysis of a larger portion of the surface, and due to the contact-less property, they can be used online.

In the literature, a large number of methods have been proposed for wear analysis using computer vision. Some study the debris which is produced due to wear (Aharoni, 1973; Vaziri et al., 1988; Benabdallah, 1997; Ledda, 2006). A survey was done by Raadnui (2005). Although studying debris helps to understand the wear process, the debris should be collected and processed separately from the specimen surface. Another possibility is to study directly the surface of the specimen itself (e.g. Kiran et al., 1998; Al-Kindi \& Shirinzadeh, 2007; Kano et al., 2008), which we apply in this paper.

Numerous works (DeVoe et al., 1992; Kiran et al., 1998; Lee et al., 2004; Al-Kindi \& Shirinzadeh, 2007) on wear investigation of a specimen surface use a postmortem analysis, which means images of the surface have been acquired at the end of testing and probably after dismounting the specimen. Although these studies reveal some aspects of the wear process, intermediate information on the wear process is missed. So, taking images during the course of wear is critical for understanding the complete wear process. However, online imaging depends on the application because installing the imaging system 
(camera, microscope, illumination) is a concern and should not affect the working condition of the system.

Semi-online studies have been performed to acquire images by stopping the machine and/or by dismounting the specimen from time to time (e.g. Tasan et al., 2005). However, stopping and resuming the experiment creates unwanted effects in the experiment, e.g. changing the temperature that may disturb the test equilibrium.

The preferred way of imaging is online, which means taking images without stopping the test rig. Some limited works have been performed by this type of monitoring. For example, in Zhang (2003) authors studied wear of specimens made from steel and aluminum at a relatively low speed $(0.5-1 \mathrm{rpm})$. They tried to detect the worn regions and estimate the wear status. To roughly follow the same locations, they used a triggering system. In Wang et al. (2000), the authors studied a specimen made from steel with a speed between 0.8 and $1.3 \mathrm{~m} / \mathrm{s}$. They tried to estimate the surface roughness, by employing a triggering system in taking images.

Another perspective in wear analysis using computer vision is the level of magnification of the surface to be studied. In some of the imaging methods, the acquired surfaces are at macro-level, leaving out the detailed information of microscopic changes (DeVoe et al., 1992; Kiran et al., 1998; Lee et al., 2004; Al-Kindi \& Shirinzadeh, 2007). Some authors applied super resolution and magnification techniques to the images (e.g. Kumar et al., 2005; Dhanasekar \& Ramamoorthy, 2008). However, these methods use off-line analysis. Even though wear scars at the macroscopic level provide evidence of the wear process, a microscopic investigation is required to understand the in-depth fundamentals of the wear process and the governing mechanisms. Furthermore, it seems reasonable that macroscopic properties of materials depend on the state and behavior of their micro-structures. Therefore, studying micro-structures may be valuable when the images are taken online. The current research trend is to use SEM, which is rather time consuming and expensive, especially for analyzing nonconductive materials (Alshibli \& Alsaleh, 2004; Artyushkova et al., 2012). Other disadvantages of SEM are ${ }^{1}$ its large size, maintenance, researcher training and image artifacts resulting from specimen preparation.

\section{Moreover}

- The installing area of SEMs should be free of any electric, magnetic, or vibration interference.

- The specimens should be in a size that fit inside the vacuum chamber.

- Working with SEMs has a small risk of radiation exposure by the electrons.

Finally, nonconductive materials (like polymers) should be coated by gold for SEM investigation. Unlike SEM, OM (optical microscopy) can be used online and in the field for condition monitoring and surface analysis.

In this paper, the focus is on micro-level wear analysis of polymers using conventional OM.

\footnotetext{
${ }^{1}$ http://www.microscopemaster.com.
}

With OMs, blur is ubiquitous due to the limited depth of field of the lens. Although blur poses a problem when acquiring high-quality images of an object, it can also be useful. Since, the amount of blurring can provide information about the distance of the surface from the plane of focus of the camera which is called depth from defocus. We explore depth from defocus to estimate the change in diameter of a polymer disc due to wear, whereas the images are obtained online using a combination of a high-speed camera and a microscope. In the conducted experiment, the rotation speed is relatively high $(200 \mathrm{rpm})$. Before applying blur estimation, the images are preprocessed to find same locations across time. This study has shown that it is possible to follow the same locations of the surface despite blurring, uneven illumination, and damage to the surface.

In this work, an online imaging system was used for wear analysis.

There have been some works on this type with relatively low speeds (Wang et al., 2000; Zhang, 2003; Kano \& Suzuki, 2009). In existing works, a triggering system to take images of the same physical location of the specimen under study was used. However, these triggering systems are not always available and cannot be used in some applications (because a triggering system needs an exact calculation to synchronize the specimen movement, illumination, and camera which may not be possible). Moreover, even with a triggering system, a misalignment is still expected (Zhang J., 2003). Here, we tried to find the same locations across time by using the image processing technique of registration.

The outline of the paper is as follows. In second section, the experimental setup is detailed. In Preprocessing section, the required preprocessing steps of the images are elaborated. These preprocessing steps include Cropping the dark corners of the images, Vignette Removal, Rough Inter-Video Registration, Stitching, and finally Exact InterVideo Registration. Applying blur/sharpness estimation methods to final images of preprocessing is described in the Blur/sharpness estimation section. We conclude and discuss future works in the Conclusion section.

\section{EXPERIMENTAL SETUP}

\section{Materials and Methods}

There are many kinds of contacting surfaces (e.g. gears, cams). In addition, different materials are used in making the contacting components (e.g. polymer, metal, steel, and aluminum) in industry. Here, our focus is on a twin-disc model of contacting, which was implemented at Laboratory Soete of Ghent University (Soleimani et al., 2012; Sukumaran et al., 2012, 2014). In this section, the experimental setup and materials used are presented. We explain the contacting model, the type of materials used in the contacting model, and the driving module along with specifications. Moreover, the imaging system is elaborated. 
The schematic of the contacting discs is shown in Figure 1. One disc is made from steel and another from polymer. Our focus has been on wear study of the polymer disc. Polymer is chosen for its dynamic wear mechanisms and transfer layer characteristics. Polymers are suitable materials to make contacting surfaces, because they are relatively cheap, easy to manufacture, and can work dry (oilless) (Rymuza, 2007).

\section{Test Rig}

The test rig used was the Forschungsstelle fur Zahnrader und Getriebebau (FZG). A schematic of the test rig is shown in Figure 2. The FZG is a modified gear oil tester, in which adaptations are done to perform experiments with nonconformal contact for pure rolling and partial sliding condition (Sukumaran, 2014).

The main components of the driving module are a gear box (single speed) and an electric motor which are connected through a belt. Both the polymer and steel discs are connected to the same gear box, so they rotate at the same speed. Applying different slip ratios is possible by using discs with different diameter for polymer and steel (the diameters of the discs are denoted by $d_{1}$ and $d_{2}$ in Fig. 1). The force between discs is applied by a dead weight through a lever. In this setup, to keep the contact between the discs even by reducing the diameter of the polymer disc, one of the shafts is mounted with a self-aligning bearing (see Fig. 2).

In summary, the following components facilitate the rolling/sliding contact:

- A single speed gear box coupled to the electric motor.

- Both discs rotate at the same speed.

- The slip ratio is attained from the difference in slip velocity. The difference in slip velocity is from the difference in diameter.

- The load used is just the dead weight mechanism.

- The arrangement maintains a continuous contact under wear.

A summary of instruments were used follows:

- The diameter change is measured using a linear variable differential transformer (LVDT) (see Fig. 2).

- The friction force and friction torque is measured online using a S-type load cell and torque transducer.

- The acquired data from the sensors were conditioned and stored in the PC using Labview 8.0.

- The sensor signals are collected using a BNC-2110 shielded connector block which in turn connected to a 16 channel card NI PCI-6036E DAQ.

For more details about the type of the sensors and their specifications, see Sukumaran (2014).

\section{Imaging System}

The microscope used was a conventional OM (Olympus BXFM-F). The camera used for high-speed imaging was an Olympus i-speed 3. The camera was connected to the microscope using an adapter with a magnification of $0.35 \times$. In the microscope itself, a $10 \times$ lens from Olympus with a numerical aperture of 0.25 was used.

Properties of the steel and polymer specimens used in online monitoring are shown in Table 1 . The discs rotated at a relatively high speed of $200 \mathrm{rpm}$. As we are interested in wear and change detection of the polymer disc, the micrographs were obtained from the polymer surface. A picture of the test setup is shown in Figure 3. The microscope was focused on the surface of the specimen at the beginning of the experiment manually and subjectively.

The dimensional changes of the specimen were collected using the stylus (LVDT). The LVDT values were used for validating the image processing results.

The diameter of the disc was $90 \mathrm{~mm}$ so its circumference was

$$
P=2 \pi r \approx 283 \mathrm{~mm} .
$$

Each image represents a band of about $0.7 \mathrm{~mm}$ width, so to take images of the whole circumference, we should take $283 / 0.7 \approx 404$ images. To be able to register images, an

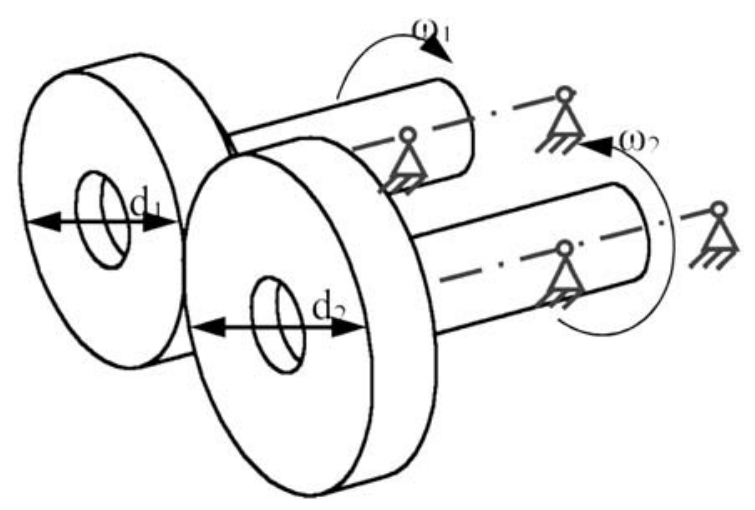

Figure 1. The schematic of the twin-disc model.

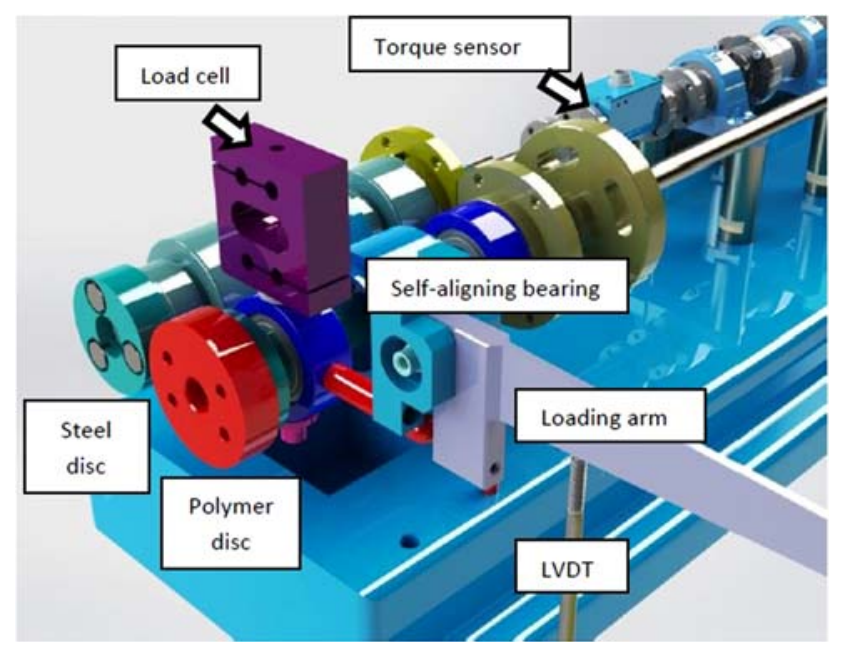

Figure 2. The schematic of the driving and load module of the test rig. LVDT means linear variable differential transformer (Sukumaran, 2014). 
Table 1. Material Properties.

\begin{tabular}{lcc}
\hline Properties & Steel & $\begin{array}{c}\text { Polymer } \\
\text { Composite }\end{array}$ \\
\hline Yield strength $(\mathrm{MPa})$ & 355 & 55 \\
Elongation (\%) & $16-20$ & - \\
Young's modulus $(\mathrm{GPa})$ & 220 & 3.3 \\
Hardness & $145 \mathrm{HV}$ & 100 Rockwell M \\
Thermal conductivity $20^{\circ} \mathrm{C}[\mathrm{W} /(\mathrm{K} . \mathrm{m})]$ & $34-45$ & 0.293 \\
Maximum allowable service & - & 130 \\
$\quad$ temperature $\left({ }^{\circ} \mathrm{C}\right)$ & & \\
\hline
\end{tabular}

overlap between successive frames is essential. For an overlap of $50 \%, 808$ images should be taken in a cycle.

On the other hand, the speed of rotation was $200 \mathrm{rpm}$, which equals 3.33 rotations/s. Thus, a rotation takes $0.3 \mathrm{~s}$. In other words, in $0.3 \mathrm{~s}, 808$ frames should be acquired which means $(808) /(0.3) \approx 2694$ frames per second (fps). Just to be on the safe side, the frame rate was set to $3,000 \mathrm{fps}$. By this frame rate, the time for recording an image was $1 / 3,000 \mathrm{~s}=333 \mu \mathrm{s}$. Therefore, the exposure time could be at most $333 \mu \mathrm{s}$. However, the shutter time was set to one-tenth of the maximum exposure time (i.e. $33 \mu \mathrm{s}$ ) to reduce the motion blur (it is possible to adjust the exposure time as a fraction of the maximum possible exposure time which in turn depends on the frame rate).

The experiment continued for $14 \mathrm{~h}$. The first video was recorded at the beginning of the rotation and after that, every $2 \mathrm{~h}$, a video was acquired. So in total, eight videos were taken at intervals of $2 \mathrm{~h}$.

\section{Preprocessing}

The plan was to create stitched images from each video, with the stitched images of different videos aligned to contain the same region of the surface. Then, the blur/sharpness estimation methods were applied to those stitched and aligned images to study the relation between the amount of the blurriness/sharpness of the images and the LVDT values. First, the corresponding frames between videos were found (rough registration). To do that, a similarity metric (explained hereafter) was employed.

After rough inter-video registration, every ten successive frames in each video were stitched. In the next step, the corresponding stitched images across time were aligned and cropped to cover exactly the same region of the surface. However, before registration and stitching, some other preprocessing steps were performed, including cropping very dark corners and vignette removal. First, these preprocessing steps are explained.

\section{Cropping}

Although a high-power external light source was used for illumination, the images were still quite dark due to short exposure time. One of the frames is shown in Figure 4a. As can be seen, the image is too dark. For better visualization, the range of pixel values of that image was increased by scaling the gray values to a whole range of $[0,255]$. The enhanced image is shown in Figure $4 \mathrm{~b}$. In this image, it can be seen that the corners of the image contain very little information. So, the images were cropped to remove some parts of corners. One of the cropped images along with its enhanced image (for better visualization) are shown in Figures $4 \mathrm{c}$ and $4 \mathrm{~d}$, respectively.

\section{Vignette Removal}

Even within the cropped region, the external light source creates severe vignetting. As seen in Figure 4d, the corners of the image are darker than central parts of the image. This uneven illumination should be compensated, otherwise it causes incorrect registration, as it is the same in both images and matching will be performed on the static vignette instead of on the surface of the specimen.

To remove the vignette, it has to be measured first. It was approximated by taking the average of all the input frames as shown in Figure 5. The vertical and horizontal black lines in the middle of the image are from the sensor of the camera (the sensor of the camera has four segments). Closely inspecting this image, some small things are also visible which are possibly due to some dust on the lens.

Having an approximation of the vignette, every frame $g(x, y)$ was divided by the vignette $v(x, y)$. Let us call the resulting image $f(x, y)$. Then the minimum and maximum of all gray values in images $f(x, y)$ were computed which we call $f_{\max }$ and $f_{\text {min }}$. Then the range of the gray scale values in each image $f(x, y)$ was increased considering $f_{\min }$ and $f_{\max }$ (i.e., the range of pixel values was mapped from $\left[f_{\min }, f_{\max }\right]$ to $\left.[0,255]\right)$. An original (cropped) frame and its vignette free frame are shown in Figures 6a and 6c, respectively. In this figure, for better vision, the range of gray values of the images in Figures $6 \mathrm{a}$ and $6 \mathrm{c}$ was increased as shown in Figures $6 \mathrm{~b}$ and $6 \mathrm{~d}$ (in the following processings, the image in Fig. $6 \mathrm{c}$ was used).

\section{Rough Inter-Video Registration}

Now that we have vignette free images, we can start to register and stitch images. First, the corresponding frames in different videos are found. By corresponding frames, we mean frames from roughly the same region of the surface. To perform that (and also for registration and stitching), a similarity metric is needed (GaudreauBalderrama, 2012). We use two similarity metrics which are explained here.

\section{Maximum Mutual Information}

Mutual information measures the amount of information a variable has about another variable (or measures the dependence between two variables) (Maes et al., 1997; 


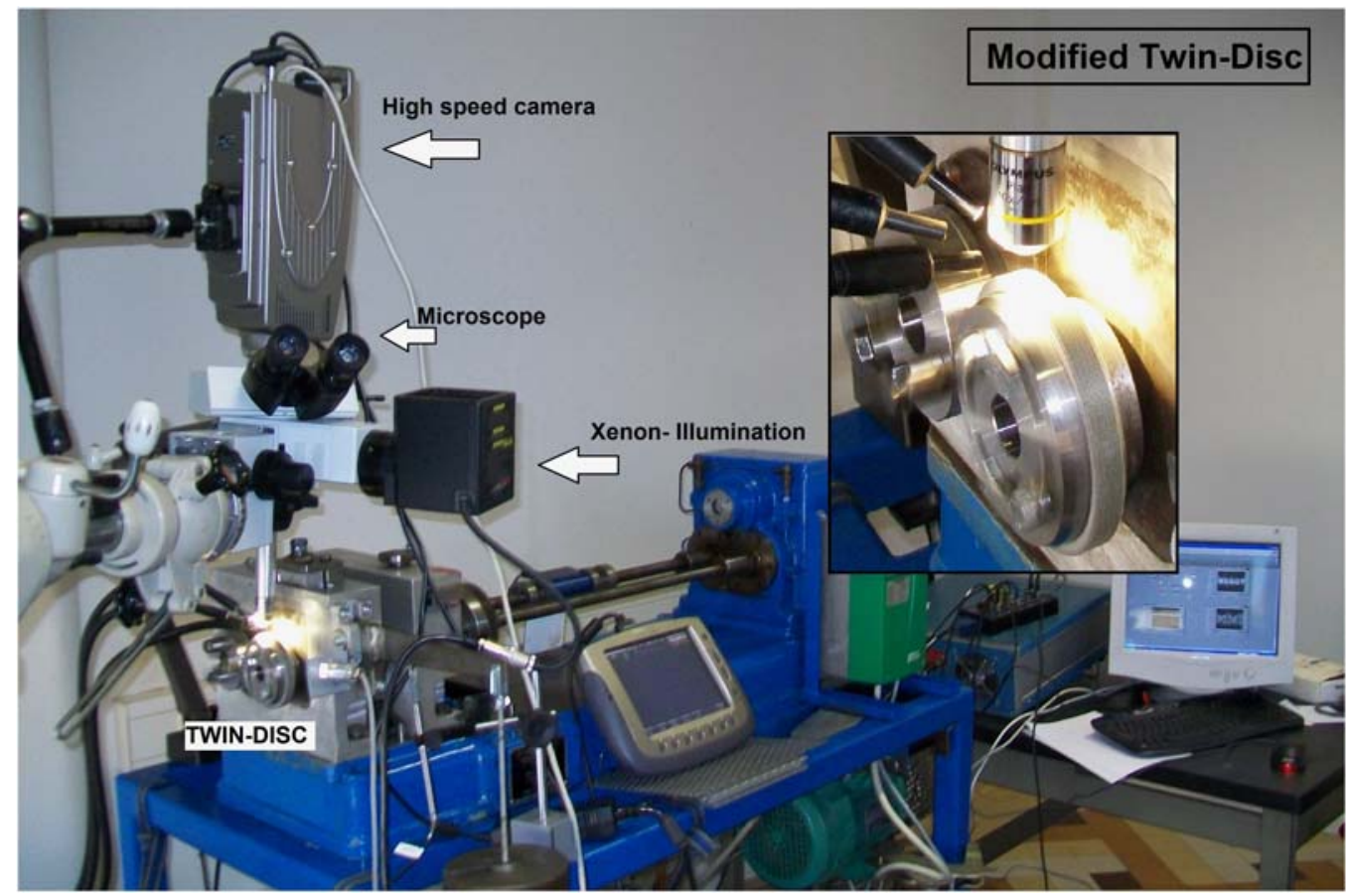

Figure 3. Test setup: the high-speed camera in conjunction with the microscope for high-speed online monitoring in a twin-disc model (Sukumaran, 2011).
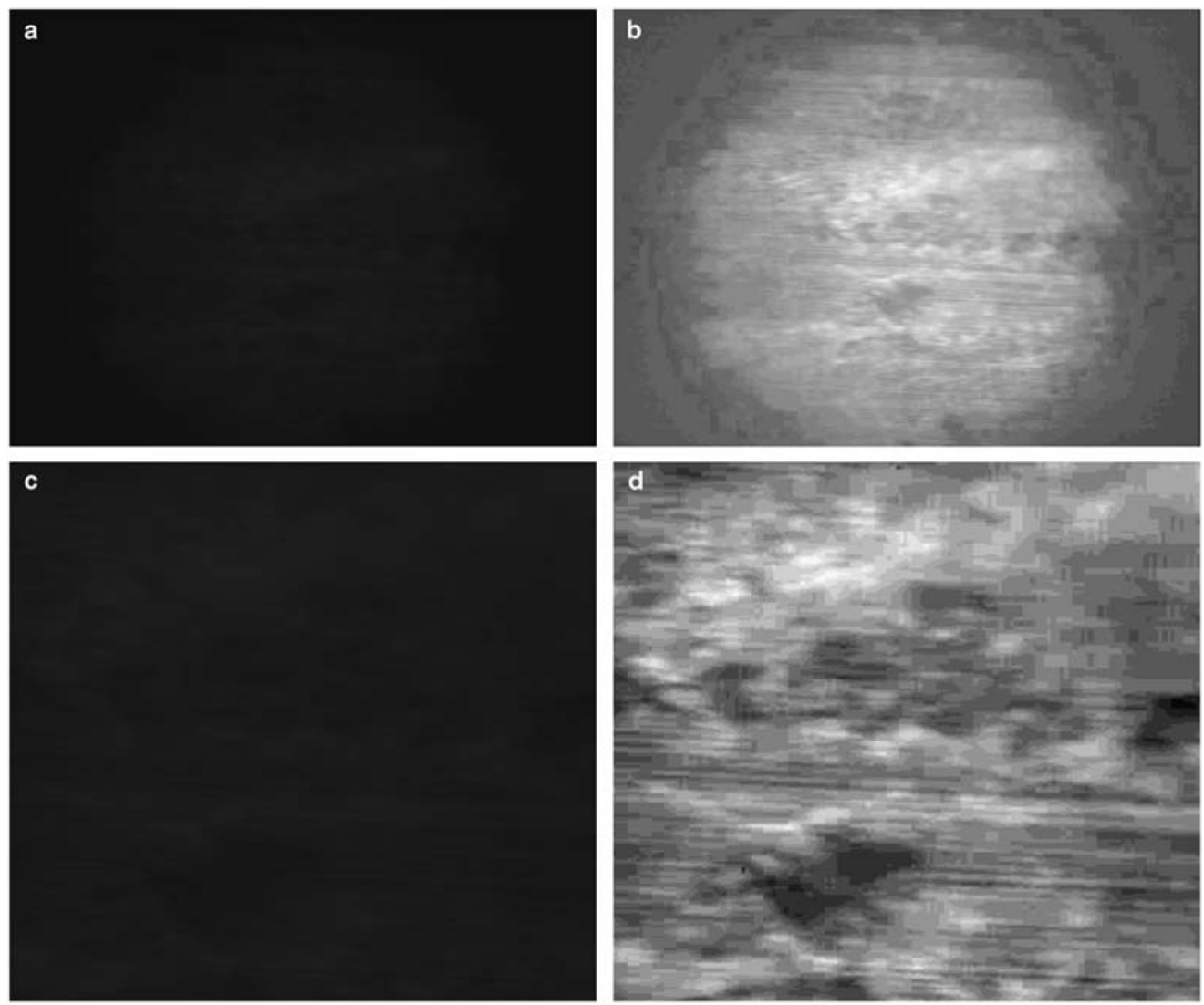

Figure 4. a: An original frame. b: The image in (a) after enhancing the contrast. c: The cropped image of image in (a). d: The image in (c) after enhancing the contrast. 
Thévenaz \& Unser, 2000). The mutual information between pixel values of two images is maximum when the images are aligned. Mutual information between two random variables

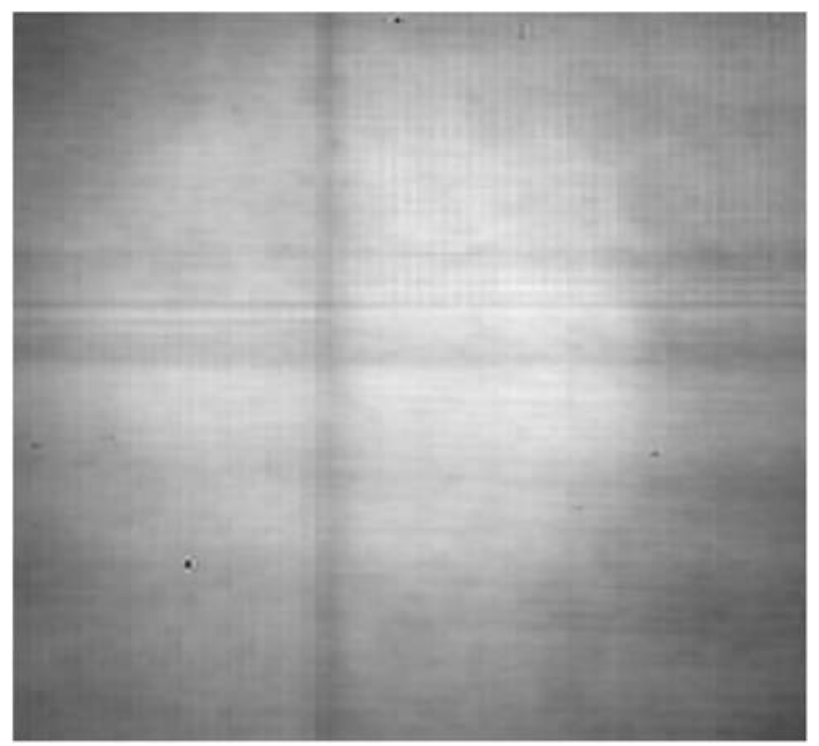

Figure 5. Approximated vignette.
$X$ and $Y$ is defined as follows:

$$
I(X, Y)=\sum_{y} \sum_{x} p(x, y) \log \left(\frac{p(x, y)}{p(x) p(y)}\right),
$$

where $p(x, y)$ is the joint probability distribution function of $X$ and $Y$, and $p(x)$ and $p(y)$ are the marginal probability distribution functions of $X$ and $Y$, respectively.

Mutual information can be equivalently expressed as

$$
I(X, Y)=H(X)+H(Y)-H(X, Y),
$$

where $H(X)$ and $H(Y)$ are the marginal entropies and $H(X, Y)$ is the joint entropy of $X$ and $Y$, where

$$
H(X)=-\sum_{x} p(x) \log _{2}(p(x))
$$

and

$$
H(X, Y)=-\sum_{y} \sum_{x} p(x, y) \log _{2}(p(x, y)) .
$$

In intensity images, the pixel values of two images being registered are considered as random variables $X$ and $Y$. The marginal and joint distributions of these random variables are estimated by obtaining normalized marginal and joint histograms of the overlapping parts of the images. Let us call the two images being registered, as "fixed" and "moving" images.
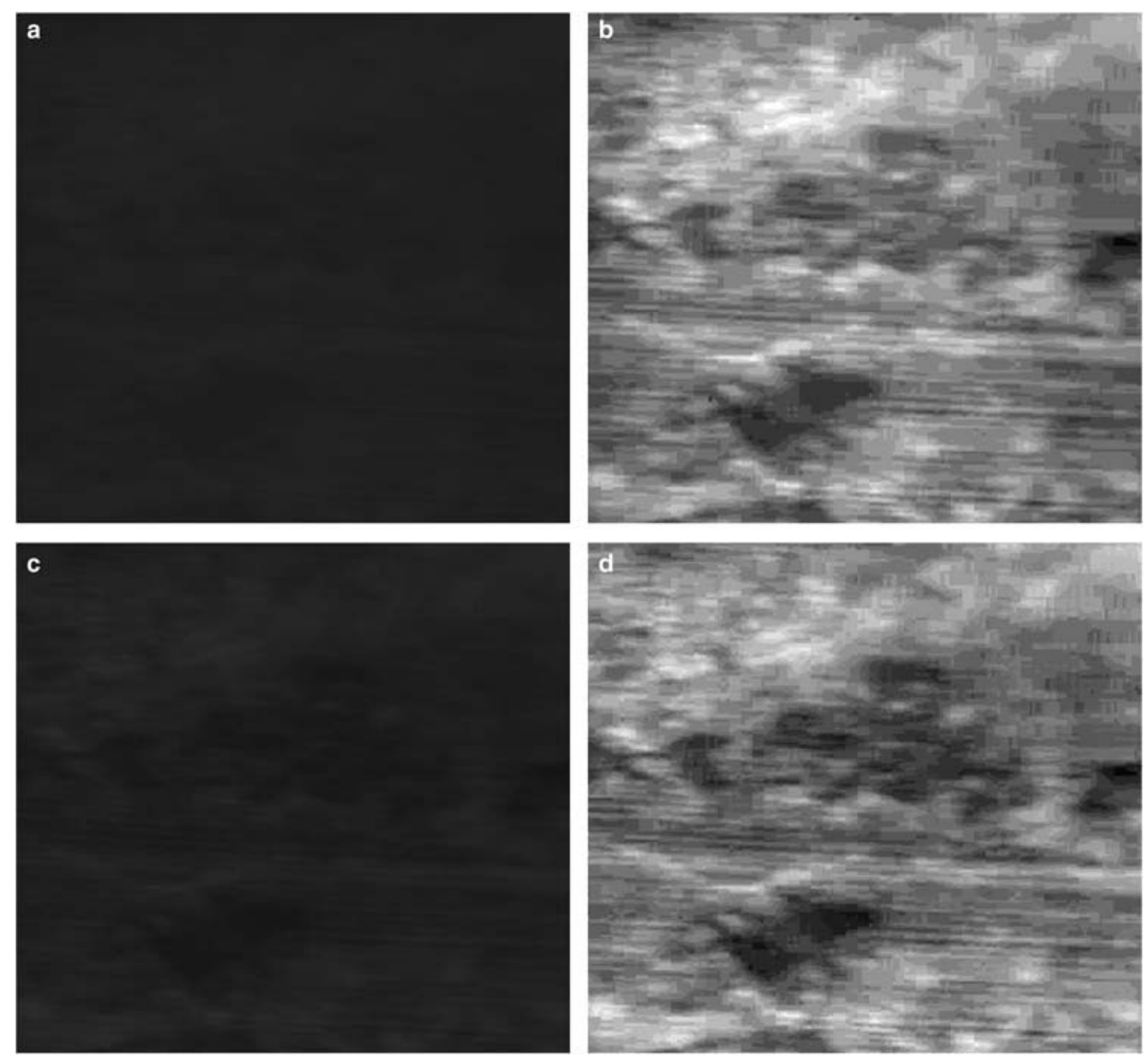

Figure 6. a: An original (cropped) frame. b: The image in (a) after increasing its gray value range. c: Frame in (a) after removing the vignette. $\mathbf{d}$ : The image in (c) after increasing its gray value range. 

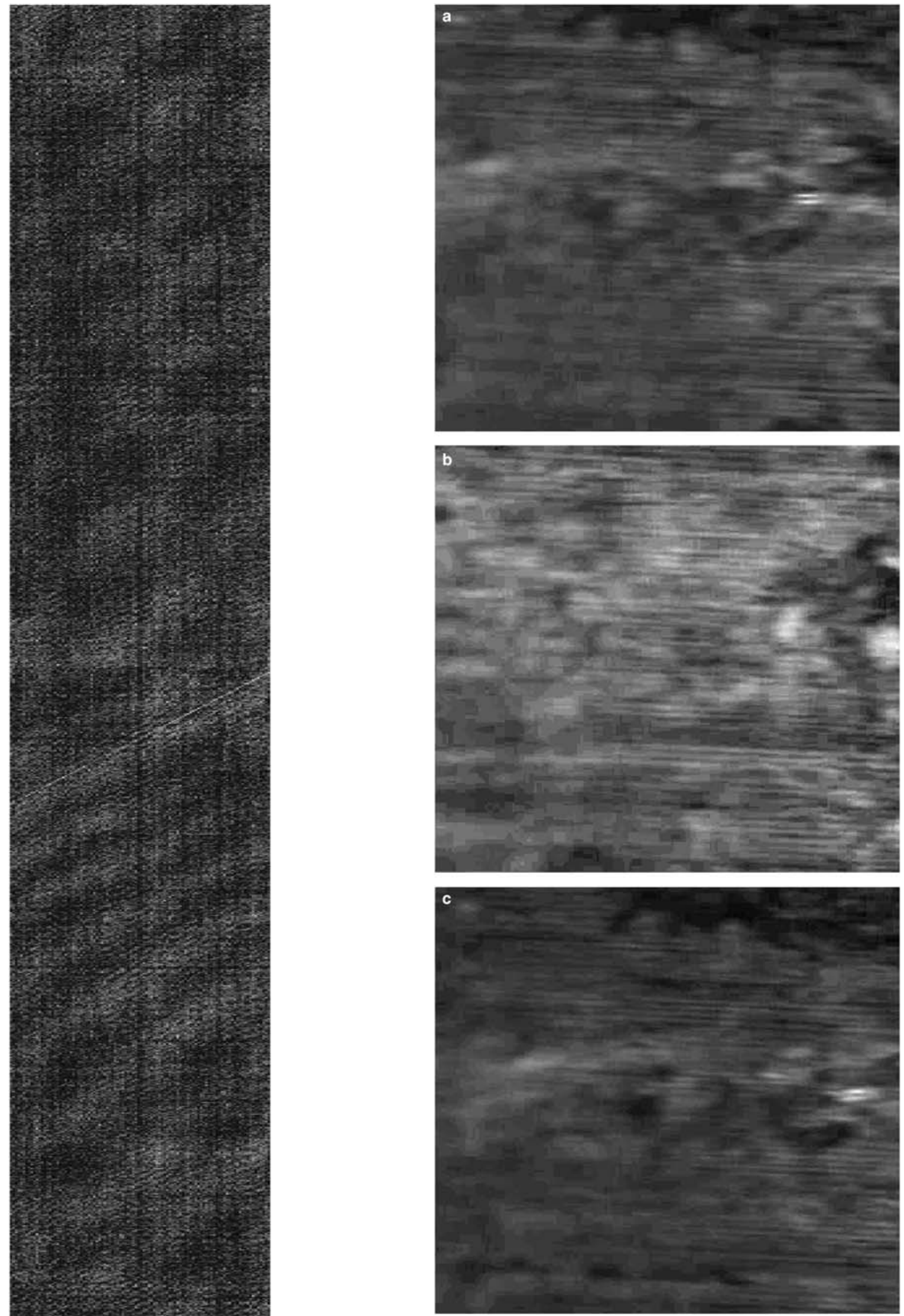

Figure 7. The phase correlations between the first 100 frames of video at hour 10 and all frames of video at hour 12 .

Figure 8. a: Frame 1 of the first video. b: Frame 198 of the second video. c: The frame 398 of the second video. 

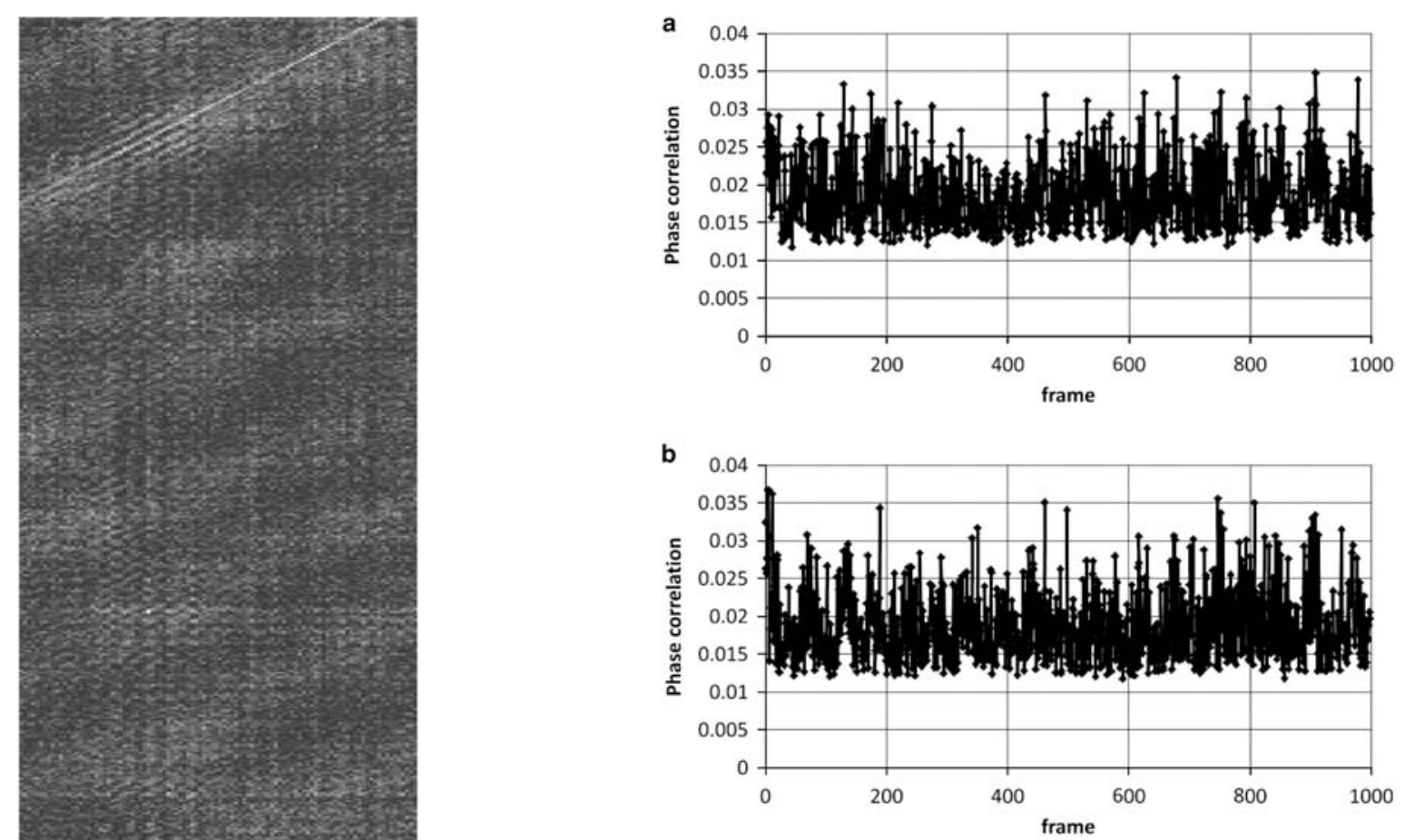

Figure 10. Maximum phase correlation between (a) frame 1 and frames 2-1,000 of the same video (maximum at 909), (b) between frame 2 and frames $3-1,000$ of that video (maximum is at 7 which is wrong).

Further, we assume that the moving image is just a translated version of the fixed image (so, there are no other types of transformation like rotation or scaling). To use the mutual information for aligning two images, the moving image is translated over the fixed image, in all possible positions, and then the mutual information of overlapping parts is calculated. The mutual information becomes maximal when the images are correctly aligned, from which the needed transform for registering the input images is derived. It should be noted that in discrete images, to align two images perfectly, one should consider sub-pixel translation as well. However, in our application we do not need such precision, and pixel-level registration is enough because we do not apply a pixel-to-pixel comparison.

The mutual information criterion works well for image registration even if the contents of the images (at overlapping parts) differs. That is why this criterion is used a lot in multi-modal registration. However, this method is rather slow. Because, the mutual information should be calculated over all possible transformations between registering images. If there is prior knowledge about the possible transformation between the two input images, one can restrict the checking situations accordingly. For example, when there is a video of an object which moves with a constant speed, the translation between all successive pairs of frames should be the same.

In our application, first we want to find corresponding

Figure 9. The phase correlations between the first 100 frames and all other frames of the same video. frames between different videos. As the misalignment between comparing frames is random, using mutual 

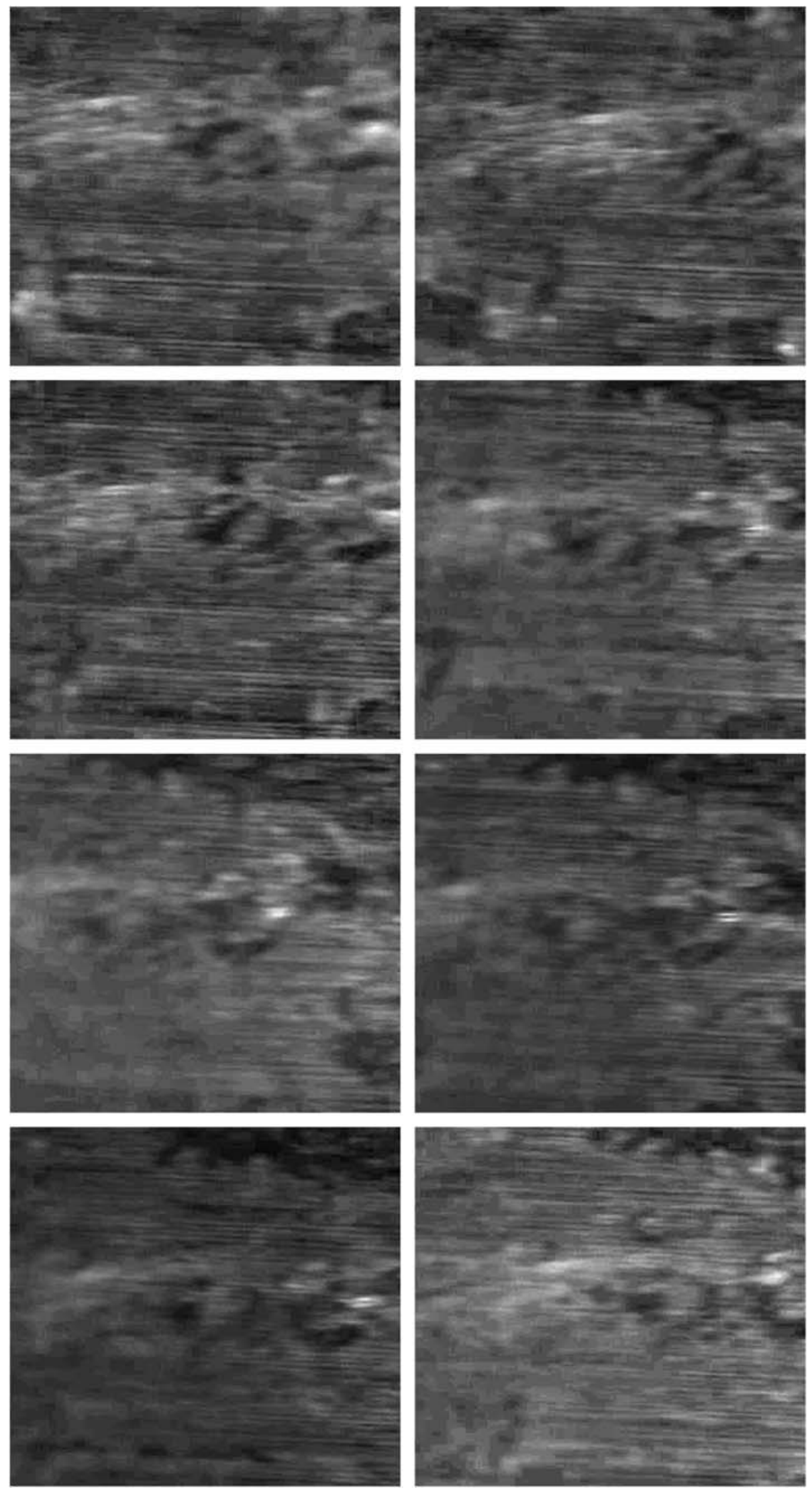

Figure 11. A set of corresponding frames of eight videos after rough registration (after enhancing their contrast). 

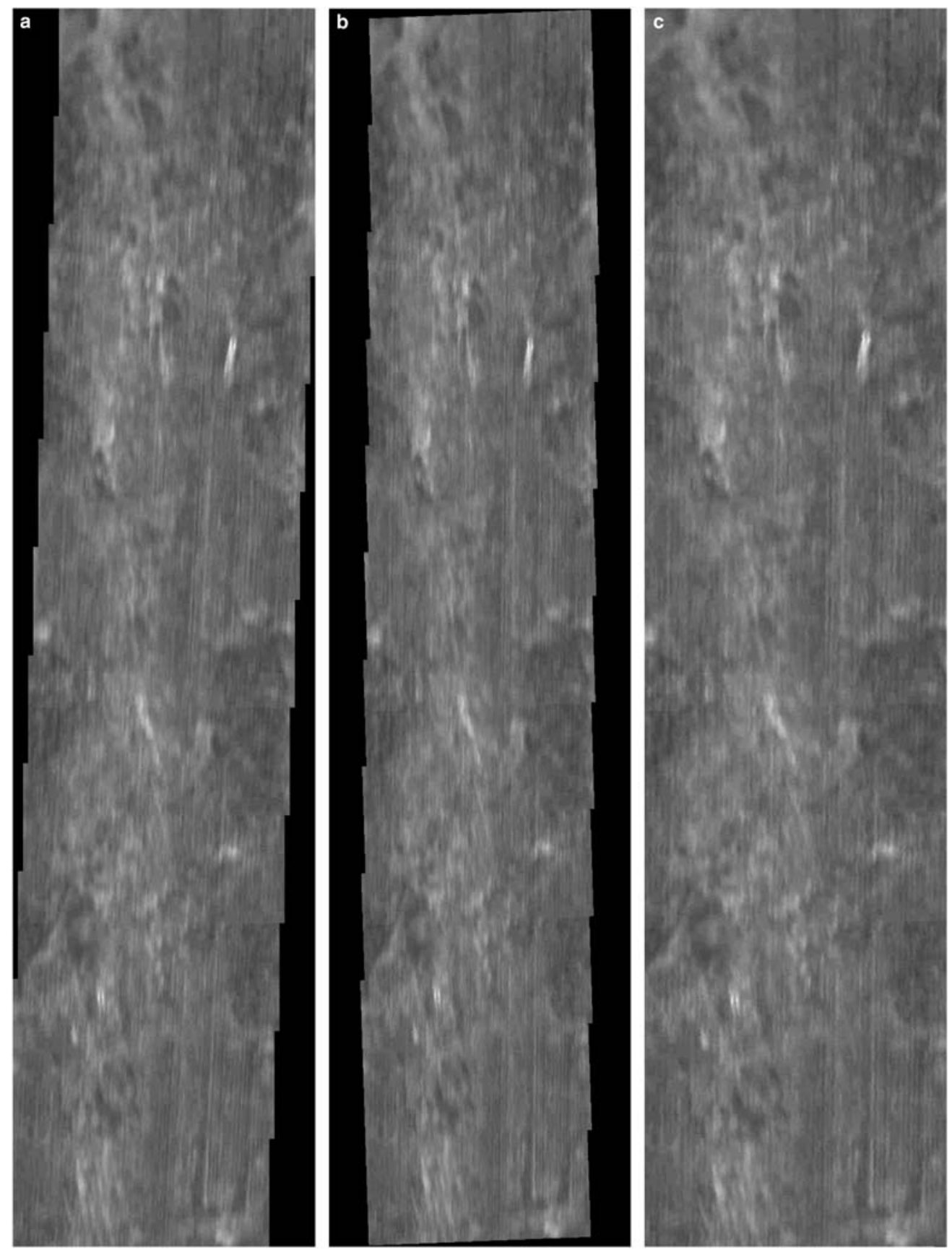

Figure 12. a: First stitched image of video 1. b: Rotated image of (a). c: Cropped image of (b).

information is very slow because all possible translations should be checked. Therefore, a faster similarity criterion, which is phase correlation, is used.

\section{Phase Correlation}

The phase correlation metric is based on the Fourier shift theorem (Ville \& Heikkila, 2007). This theorem states that if two images $f_{1}$ and $f_{2}$ differ only by translation $\left(x_{0}, y_{0}\right)$, i.e.

$$
f_{2}(x, y)=f_{1}\left(x-x_{0}, y-y_{0}\right)
$$

then, their Fourier transforms $F_{1}$ and $F_{2}$ has the following relation:

$$
F_{2}(u, v)=F_{1}(u, v) \exp \left(-i\left(u x_{0}+v y_{0}\right)\right)
$$

The above equation means that the images have the same Fourier magnitude, whereas their phase difference is related to their spatial displacement. If the both sides of equation (4) are multiplied by $F_{1}^{*}(u, v)$ and then divided by $\left|F_{2}(u, v) F_{1}^{*}(u, v)\right|$, where ${ }^{\star}$ denotes complex conjugate, and 


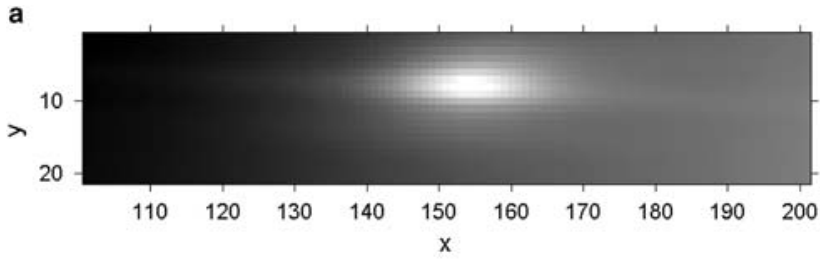

b

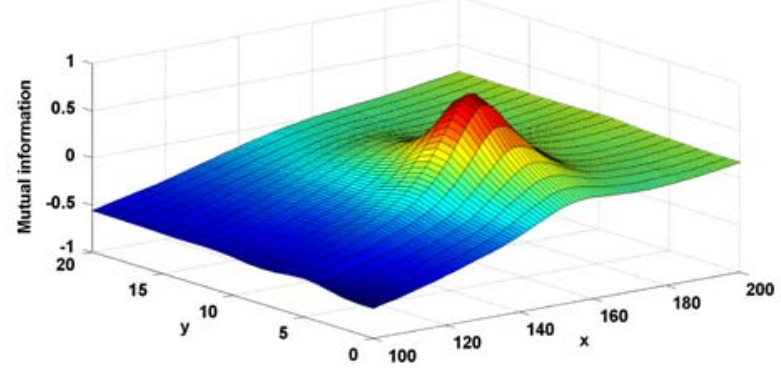

Figure 13. Mutual information between two first images of video 1: (a) as an image, (b) as a surface.

I.I is the magnitude, we will have

$$
S(u, v)=\frac{F_{2}(u, v) F_{1}^{*}(u, v)}{\left|F_{2}(u, v) F_{1}^{*}(u, v)\right|}=\exp \left(-i\left(u x_{0}+v y_{0}\right)\right)
$$

In deriving the above equation, the following equalities are used:

$$
F_{1}(u, v) F_{1}^{*}(u, v)=\left|F_{1}(u, v)\right|^{2} \text { and }\left|F_{2}(u, v)\right|=\left|F_{1}(u, v)\right| .
$$

The inverse Fourier transform of the right side of equation (5) is $\delta\left(x_{0}, y_{0}\right)$, which means a $\delta$ dirac function at $\left(x_{0}, y_{0}\right)$. So, the location of the spike in the inverse Fourier transform of $S(u, v)$ in equation (5) shows the translation between the two images. However, this is the ideal case. Because in image registration, the contents of the input images are the same in some regions and are different in other regions. So, the moving image is not just the translated version of the fixed image. Moreover, in our application, even the content of the same regions may be changed due to wear and blurring. So, this similarity criterion may not work in some situations. However, we could exploit it to find corresponding frames between two videos by calculating the phase correlation between a set of successive frames of a video with all other frames of another video. By this strategy, one can be sure that errors are detected.

For example in Figure 7, the phase correlation between 100 frames of a video with all other 1,000 frames of another video is shown as an image. A line with a slope of $45^{\circ}$ is visible in this image, which shows the correspondence point between two videos. If we just take the phase correlation between the first frame of the first video and all the frames of the second video, we will see that the maximum correlation is for frame 198 of the second video. However, from the line it is found to be 398 which is the correct one by inspecting frames subjectively. In Figure $8 a-8 c$, frame 1 of the first video and frames 198 and
398 of the second video are shown, respectively (after increasing the contrast by increasing the range of gray values to the range $[0,255])$.

The phase correlation criterion can also be used to determine if a video contains pictures of a whole circumference. The phase correlations between the first 100 frames of a video with all next frames of that video are shown as an image in Figure 9. A line with slope of $45^{\circ}$ is visible in this image (on the top of the image), which reflects the location of the wrapping point (the wrapping point is the frame that a whole cycle of the surface is completed starting from the first frame of the video). It is frame 909 for the first frame. It means that frame 908 is the most similar frame. At a rotation speed of $200 \mathrm{rpm}$ and 3,000 fps, we should theoretically get the same image after 900 frames $((60 \mathrm{~s} \times 3,000 \mathrm{fps}) / 200 \mathrm{rpm}=900$ frames $)$. This indicates that the rotation speed was slower than $200 \mathrm{rpm}$. So, as a corollary of this study, computer vision can detect small errors in mechanical devices.

Here, we illustrate why to find the wrapping point of a video (or for finding the corresponding frames between two videos) we calculated the phase correlation of several frames of a video with all other frames of that video. The phase correlations between frame 1 of a video and frames $2-1,000$ of that video are plotted in Figure 10a. In this plot, the maximum value is at 909 which is correct. However, it is not always correct. For example in Figure 10b, the phase correlations between frame 2 and frames $3-1,000$ of that video are plotted. In this case, the maximum is located at 7 , whereas it should be at $910(2+908)$. The value at 910 (i.e., correct correspondence) is the ninth largest value. Moreover, the maximum phase correlation makes a mistake in finding the corresponding frames of frames 3,4 , and 5 . So, using phase correlation between just 1 frame and all other frames can easily cause mistakes.

For illustration, a set of the corresponding frames of eight videos after rough registration are shown in Figure 11 (after enhancing their contrast). The next step is stitching of the frames of each video.

\section{Stitching}

In our application, in addition to misalignment of successive frames of a video, the content of images (frames) are also subject to differ due to change of the surface and blurring. So the phase correlation criterion is not suitable. However, maximum mutual information is expected to work well in this situation. We mentioned earlier that if all possible translations between two input images of registration (stitching) are checked, the method is very slow. However, here we know that there is only a translation between successive frames. We also know that the translations between two successive frames are more or less similar because the speed of the rotation is constant. So, we just check a small range of possible translations. We calculated the mutual information for vertical translation between 0 and 20 pixels (because the moving image translated up in comparison with the first image in this range), and horizontal translation 

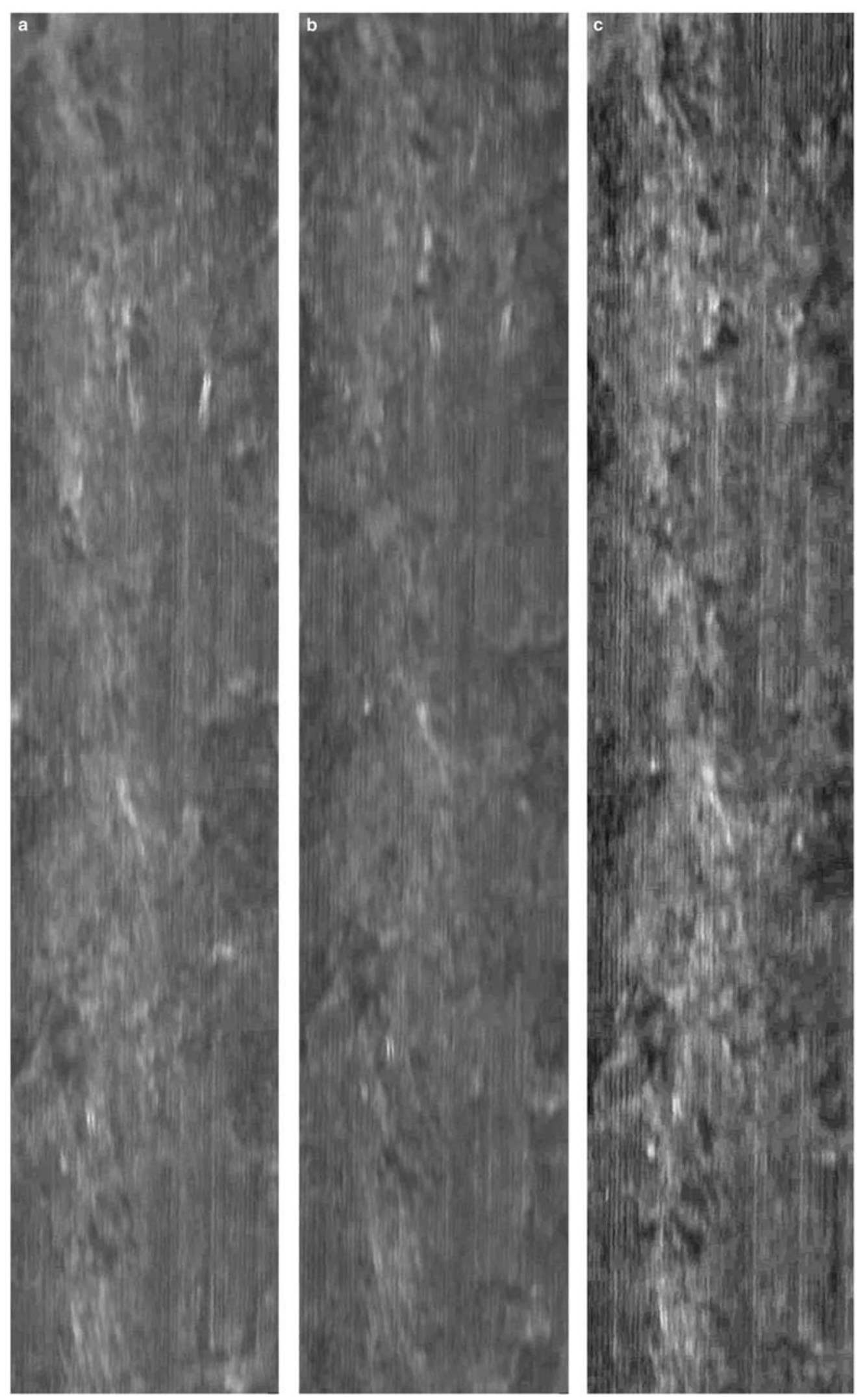

Figure 14. The first stitched images of videos at (a) hour 0, (b) hour 2, (c) hour 4. 


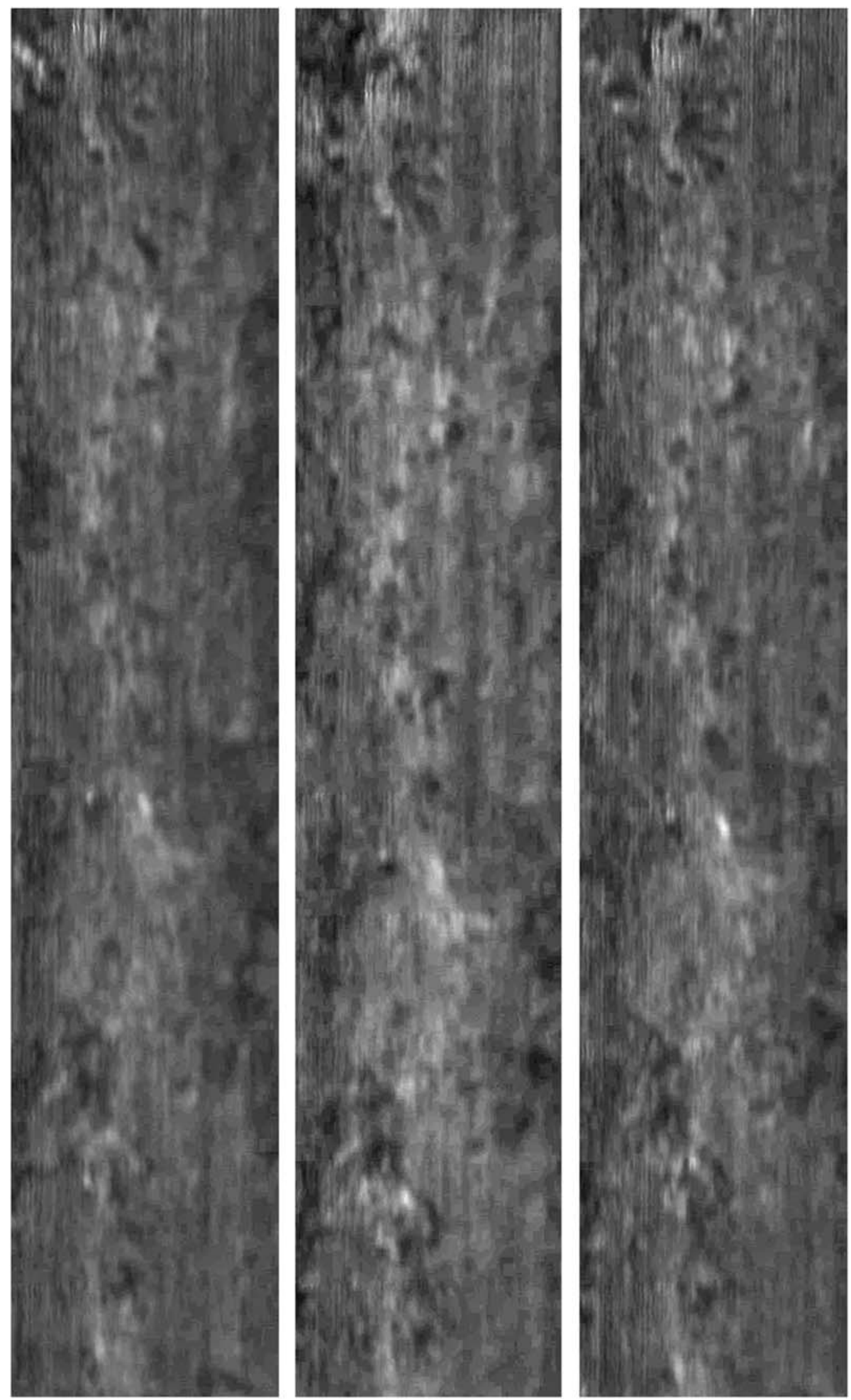

Figure 15. The first stitched images of videos at hours 6,8 , and 10 . 
between 100 and 200 pixels (because the moving image was translated left in comparison with the fixed image in this range).

One of the stitched images is shown in Figure 12a (the first frame on the bottom). As can be seen in this image, the stitched image does not contain a rectangular part of the surface. It means that the camera was rotated a bit while looking at the specimen. The stitched images should be cropped to remove dark areas. However, to reduce cropped regions, we rotated the stitched images about $2^{\circ}$ degrees (calculated based on the angle between the border of the stitched images and the border of the surface in the stitched images). The image in Figure 12a is rotated and shown in Figure $12 \mathrm{~b}$. Finally, the rotated images were cropped to remove dark regions. The cropped image of the image in Figure $12 \mathrm{~b}$ is shown in Figure 12c.

For illustration, the computed mutual information [equation (2)] between first two images of video 1 is shown as an image in Figure 13a and as a 3D surface in Figure 13b. Here, the mutual information is just calculated for $x$ (horizontal translation) between 100 and 200 and $y$ (vertical translation) between 0 and 20. In this case, the maximal mutual information is located at $(x, y)=(153,7)$.

The first stitched images of eight videos (after rotation and cropping) are shown in Figures 14 to 16, respectively (the contrast of images increased by mapping the range of gray values to $[0,255])$.

\section{Exact Inter-Video Registration}

The final step before applying blur/sharpness estimation is to register and crop stitched images to cover the same area of the surface. The length of stitched images is at least 1,700 pixels, whereas the height is 338 pixels. To reduce computational cost in calculating the mutual information, we just took the left part of images with length of 700 pixels (and whole width of the images).

Having the translation information between corresponding stitched images (using maximum mutual information), they were cropped to cover the same area of the surface. One of the final sets of registered and cropped stitched images is shown in Figures 17 to 19.

In registering stitched images, we found that sometimes the global maximum of mutual information does not reflect the correct translations. For example, the mutual information between two stitched images taken at hours 2 and 4 is shown in Figure 20a as an image and in Figure 20b as a 3D surface (the input images of this registration are shown in Fig. 14b and 14c). In the computed mutual information (which was computed for $x=0-100$ and $y=0-30$ ), there are three local maxima with mutual information of $-3.9,-3.3$, and -3.7 located at $(x, y)=(18,15),(92,14)$, $(100,30)$, respectively. The third local maximum is located at the border which we ignore, as our observations show that the maxima of mutual information at the borders usually give wrong transformations. Between two other local maxima, the second one is larger (it is the global maximum
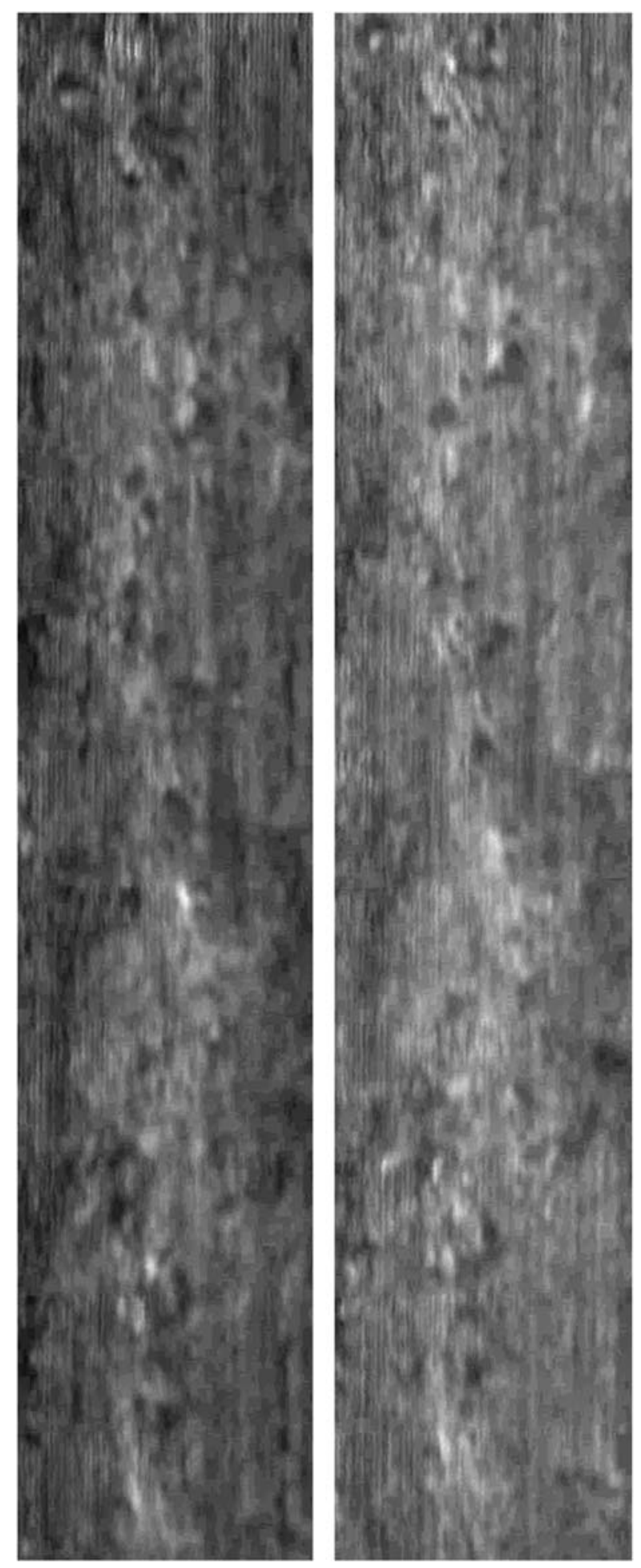

Figure 16. The first stitched images of videos at hours 12 and 14 .

of the mutual information). However, the location of this maximum is $(x, y)=(92,14)$, which states that the horizontal translation between two images is 92 pixels and the vertical translation is 14 which is not correct based on our inspection. The correct translation info is obtained from the local maximum at $(x, y)=(18,15)$. This error is probably due to the texture nature of the images. So, to use 


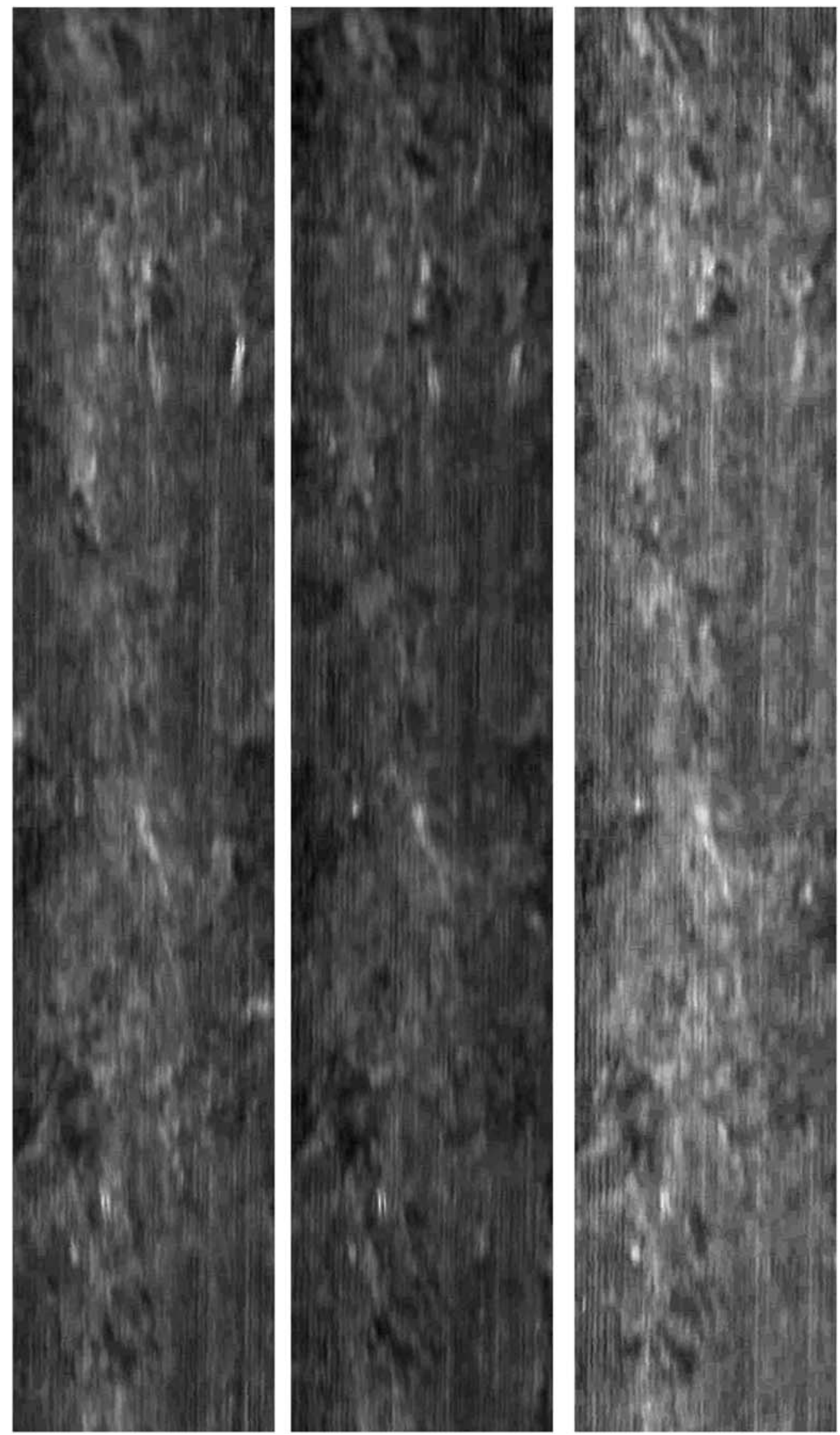

Figure 17. A set of registered and cropped stitched images: for hours 0,2 , and 4 . 

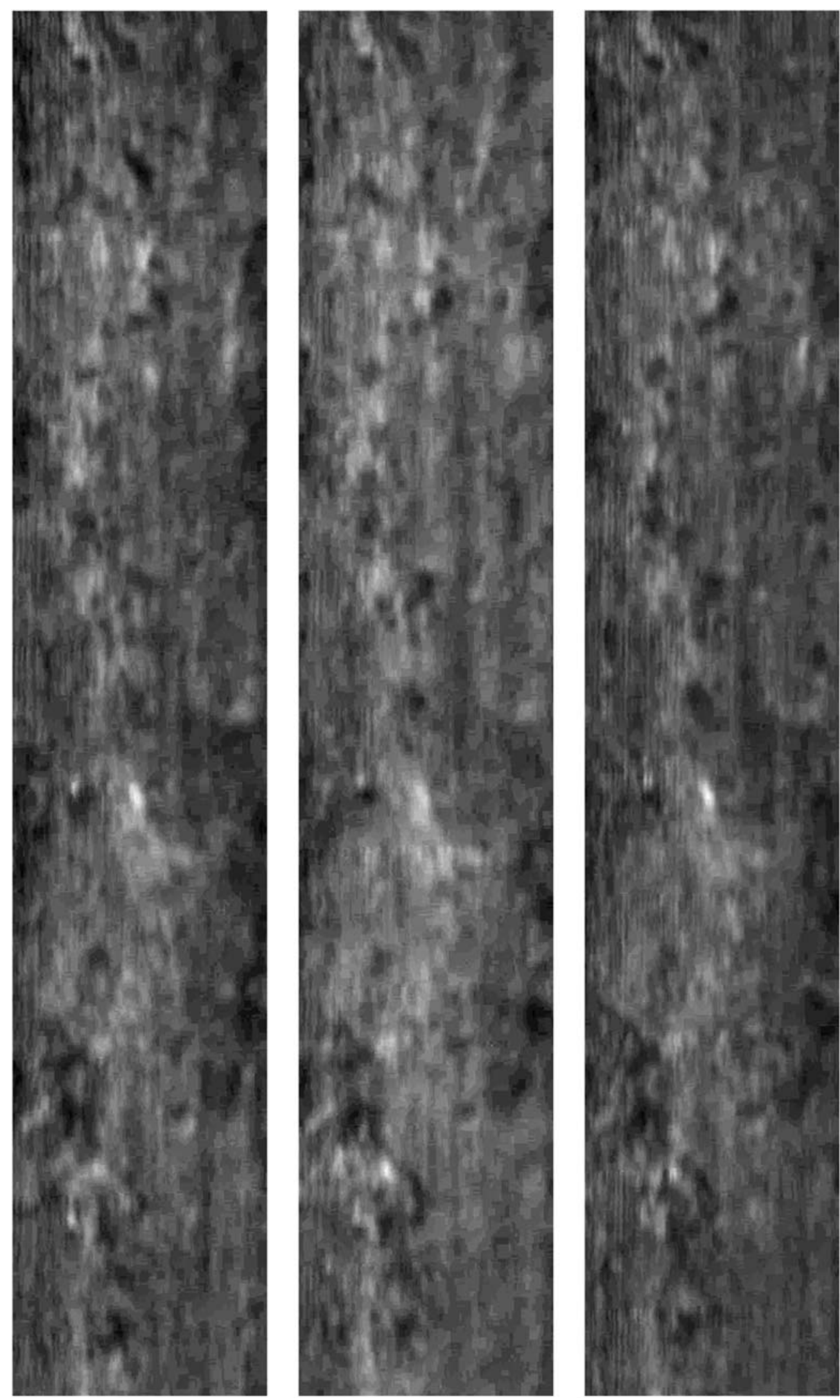

Figure 18. A set of registered and cropped stitched images: for hours 6, 8, and 10. 

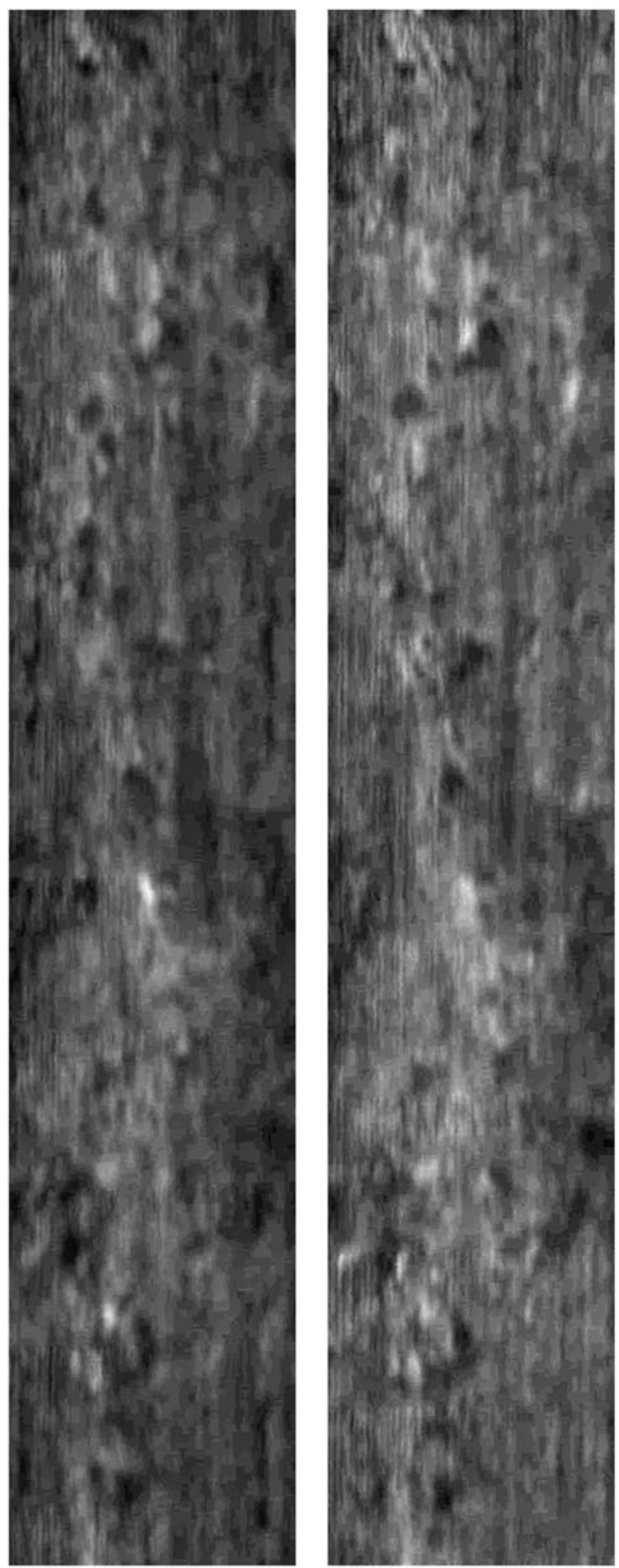

Figure 19. A set of registered and cropped stitched images: for hours 12 and 14.

mutual information for registering this type of images, extra control or prior knowledge about the possible amount of misalignment is necessary. In our application, the translations between each pair of stitched images are more or

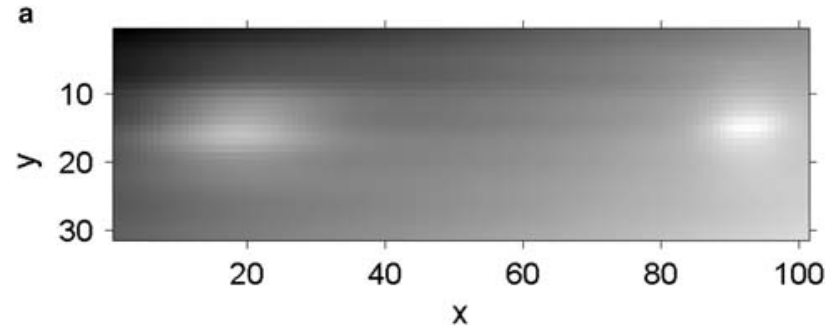

b

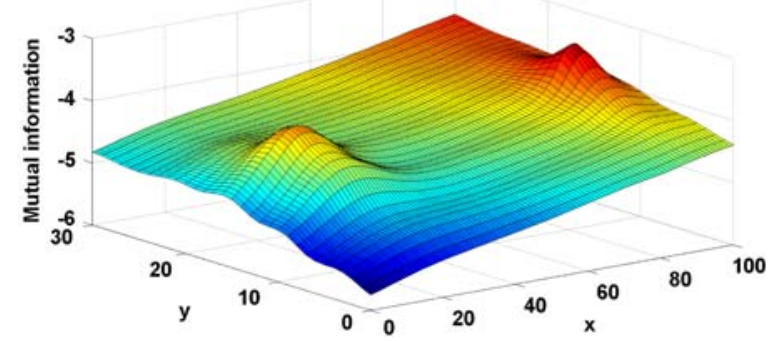

Figure 20. The mutual information between two stitched images from hours 2 and 4: (a) as an image, (b) as a 3D surface.

less the same. So, the translations which are far from the average show the errors.

\section{Blur/Sharpness Estimation}

Using the processing steps of previous sections, we created five sets of stitched images, each covering the same region of the specimen across time. Then, the estimated sharpness values of the five sets of images using 18 methods (Soleimani et al., 2013; Soleimani, 2014) were calculated. Afterwards, the average of the sharpness of the images from the same time along with their standard deviations are computed and shown in Figures 21 to 23. The LVDT values associated with the times of recording videos are shown in Table 2 and plotted in Figure 23d.

As can be seen in Figure 21d, the Spectrum method produced small error bars. From the curve for this method, it can be seen that

- Fact (a): the images at hour 6 are more blurred than the images at hour 4.

- Fact (b): the images at hour 10 are sharper than the images at hour 8.

- Fact (c): the images at hour 4 are more blurred than the images at hour 2.

From the curve in Figure 22a, which is for the Marziliano method, facts (a) and (c) are confirmed.

From the curves in Figures 21e to 21g, 22c, 22e and 23b and the methods Riemanian, NSS, Cogarc24, Laplacian, and Gradient, respectively, it is clear that facts (a) and (b) are confirmed.

From the curves in Figures $21 \mathrm{~h}$ and 22b, 22d, 22f, 22g, 22h, which are for the methods Nrjpeg, Marichal, Cogarc34, Auto, Kautsky and Jnbm, fact (a) is confirmed. 

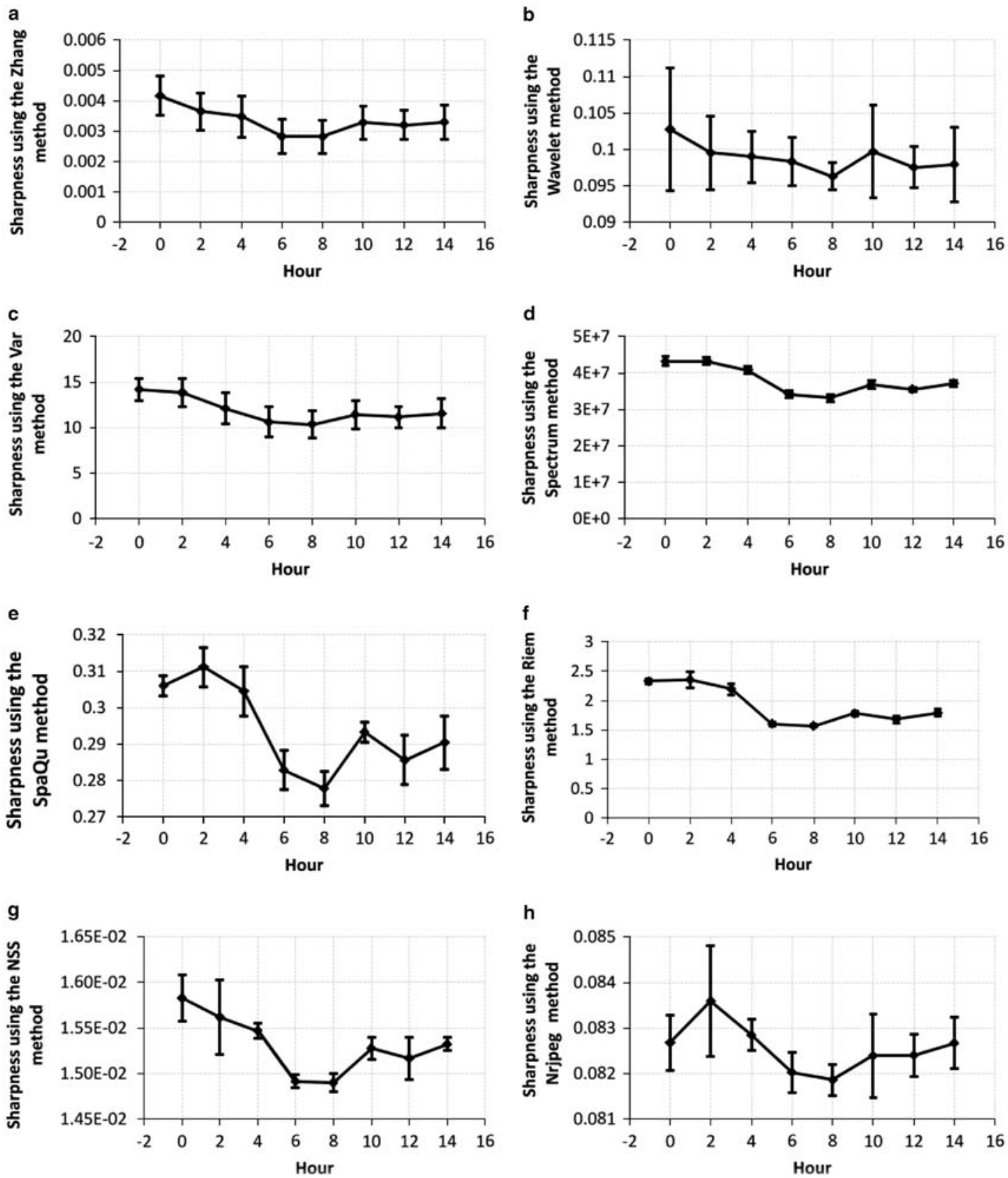

Figure 21. The average and the standard deviation of sharpness of five sets of images using different methods: (a) Zhang method, (b) Wavelet method, (c) Var method, (d) Spectrum method, (e) SpaQu method, (f) Riem method, (g) NSS method, (h) Nrjpeg method.

From the curves in Figures 21a, 21b, 21c and 23a, 23c, which are for the methods of Zhang, Wavelet, Var, Hp, and Cpbd, none of the facts can be confirmed. It means that these methods are unreliable for this application.

By subjective inspection of the stitched images (one set of stitched images is shown in Figs. 17-19), one can confirm the following statements:

- In all five sets, the images at hour 6 are more blurred than images at hour 4 [fact (a)].

- At least in three cases, the images at hour 10 are sharper than images at hour 8 [fact (b)].
- In three sets, the images at hour 4 are more blurred than images at hour 2 [fact (c)].

- At least in three sets, the images at hour 12 are more blurred than images at hour 10.

- In two sets, the images at hour 14 are sharper than images at hour 12.

The LVDT values are shown in Table 2 and are plotted in Figure 23d. The change of LVDT values between successive time sampling are also shown in Table 2 . By comparing the change in LVDT values and the esti- 

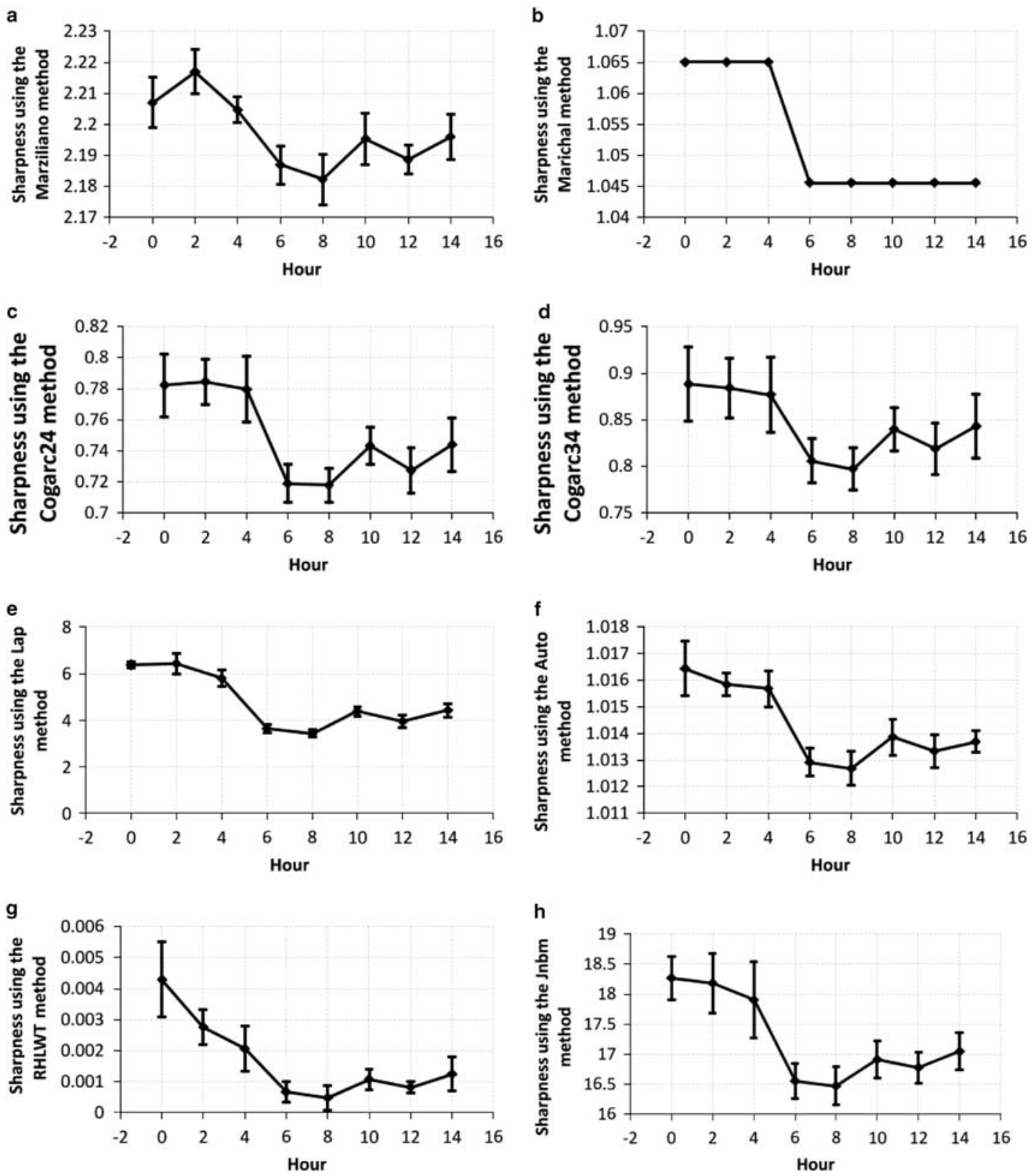

Figure 22. The average and the standard deviation of sharpness of five sets of images using different methods: (a) Marziliano method, (b)Marichal method, (c) Cogarc24 method, (d) Cogarc34 method, (e) Lap method, (f) Auto method, (g) RHLWT method, (h) Jnbm method.

mated sharpness values, the following conclusions can be drawn:

- The amount of change of LVDT values between hours 4 and 6 is $2.9 \mu \mathrm{m}$. Most blur estimation methods present a significant change between the sharpness at images of these two times [fact (a)].

- The amount of change of LVDT values between hours 8 and 10 is $1.2 \mu \mathrm{m}$. Most blur estimation methods present a significant change between the sharpness of images at these two times [fact (b)].
- The amount of change of LVDT values between hours 2 and 4 is $1.8 \mu \mathrm{m}$. Two methods (Spectrum and Marziliano) present a significant change between the sharpness at images of these two times [fact (c)].

- The amount of change of LVDT values between hours 6 and 8 , between hours 10 and 12, and between 12 and 14 are $0.3,0.5$, and $0.7 \mu \mathrm{m}$, respectively . No blur estimation method present a significant change between the sharpness of images of these times.

- The amount of change of LVDT values between hours 0 and 2 is $1.4 \mu \mathrm{m}$, whereas no blur estimation method 
Table 2. Linear Variable Differential Transformer (LVDT) Values in Micron.

\begin{tabular}{lccccccc}
\hline Hours & 0 & 2 & 4 & 6 & 8 & 10 & 12 \\
\hline LVDT & 0.0 & -1.4378 & -3.2462 & -0.3122 & -0.0312 & 1.1345 & 0.6316 \\
LVDT change & - & 1.4 & 1.8 & 2.9 & 0.3 & 1.2 & 0.5 \\
\hline
\end{tabular}

present a significant change between the sharpness of images of these two times.

In summary, in three out of four cases the change in LVDT values is $>1.2 \mu \mathrm{m}$, some blur estimation methods present a significant change between blur/sharpness values of images at those times. The only case that the change in LVDT values is $>1.2 \mu \mathrm{m}$, whereas no blur estimation method presents a significant change in blur/sharpness values is between times 0 and 2 . It can be explained by the fact that at the beginning of the experiment, focusing of the imaging system on the surface was performed subjectively which is limited by human vision.

In summary, the best method (in agreement with LVDT values) is the Spectrum method. Seven methods are in the second position.

The results show that it may be possible to detect change of the diameter of the specimen $>1.2 \mu \mathrm{m}$ using computer vision. However, more experiments are required to deduce a strong claim. Moreover, to understand whether the change is increasing (due to thermal expansion) or decreasing, extra information is needed because the images of the surface located at two different distances produce the same blur/sharpness value. These two distances are located equally on both sides of the sharpest position.

\section{CONCLUSION AND FUTURE WORK}

We studied the possibility of using computer vision for change detection of a specimen made from polymer without stopping the rotation at the micro-level. Despite changes in the surface, blurring and uneven illumination, it is possible to track the same locations in the images. It is shown that some blur/sharpness estimation methods can detect change in the diameter of the specimen with precision of about $1.2 \mu \mathrm{m}$. However, more experiments are required to confirm this ability. Moreover, extra information should be entered in the analysis to know the direction of the change in the diameter of the disc (increasing or reduction).

In this experiment, we acquired images of the whole surface, however, using a triggering system, one can acquire images from more and less a same part (e.g., 60 images of a same region) instead of 1,000 images of whole circumference. By this strategy, the processing time decreases.

\section{ACKNOWLEDGMENTS}

This investigation was supported and funded partially by a scholarship from Ministry of Science, Research and
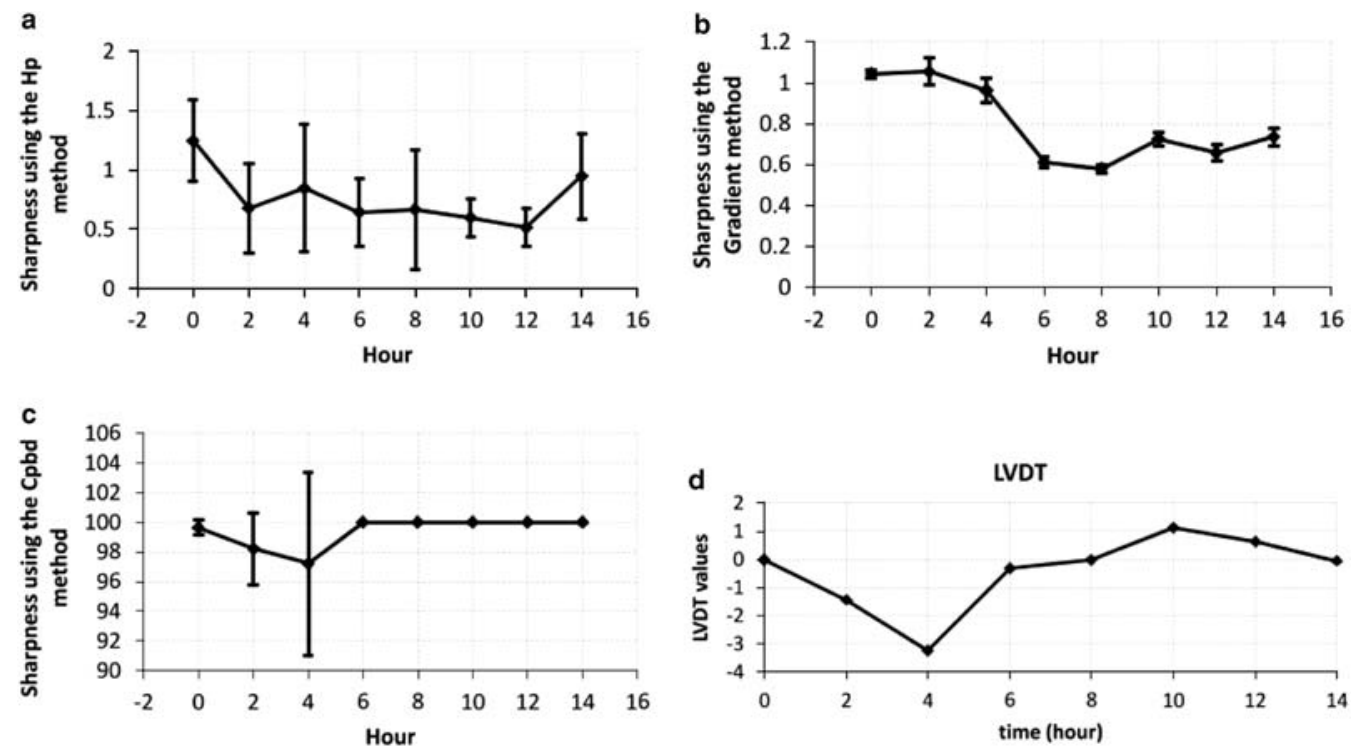

Figure 23. a-c: The average and the standard deviation of sharpness of five sets of images using different methods. d: The linear variable differential transformer (LVDT) values. 
Technology of Islamic Republic of Iran, and partially by the FWO (FondsWetenschappelijkOnderzoek ${ }^{2}$ ) Research of Belgium (FWO Grant no. G066908).

\section{REFERENCES}

Aharoni, S. (1973). Wear of polymers by roll-formation. Wear 25(3), 309-327.

Al-Kindi, G. \& Shirinzadeh, B. (2007). An evaluation of surface roughness parameters measurement using vision-based data. Int J Mach Tool Manu 47(3), 697-708.

Alshibli, K.A. \& Alsaleh, M. (2004). Characterizing surface roughness and shape of sands using digital microscopy. ASCE, J Comput Civ Eng 18(1), 36-45.

Artyushkova, K., Svitlana, P., Madhu, D. \& Plamen, A. (2012). Use of digital image processing of microscopic images and multivariate analysis for quantitative correlation of morphology, activity and durability of electrocatalysts. RSC Adv 2, 4304-4310.

BAYER, R.G. (2004). Mechanical Wear Fundamentals and Testing, Revised and Expanded. New York: CRC Press.

Benabdallah, H. (1997). Reciprocating sliding friction and contact stress of some thermoplastics against steel. J Mater Sci 32(19), 5069-5083.

DeVoe, D., Knox, L. \& Zhang, G. (1992). An experimental study of surface roughness assessment using image processing. Technical report. Systems Research Center, University of Maryland, College Park.

Dhanasekar, B. \& Ramamoorthy, B. (2008). Assessment of surface roughness based on super resolution reconstruction algorithm. Int J Adv Manuf Tech 35(11), 1191-1205.

Gaudreau-Balderrama, A. (2012). Multi-Modal Image Registration. Boston: Boston University.

Kano, S., Homma, H., Sasaki, S. \& Shimura, H. (2008). In situ monitoring of friction surfaces and their sequence pattern analysis. Philos Trans A Math Phys Eng Sci 366(1865), 665-671.

KANO, S. \& SUZUKI, T. (2009). In-situ monitoring of friction surfaces and friction modelling by surface pattern analysis. Wear 267(5), 1075-1079.

Kiran, M., Ramamoorthy, B. \& Radhakrishnan, V. (1998). Evaluation of surface roughness by vision system. Int J Mach Tool Manu 38(5), 685-690.

Kumar, R., Kulashekar, P., Dhanasekar, B. \& Ramamoorthy, B. (2005). Application of digital image magnification for surface roughness evaluation using machine vision. Int J Mach Tool Manu 45(2), 228-234.

LEDDA, A. (2006). Mathematical morphology in image processing. $\mathrm{PhD}$ thesis, Ghent University, Ghent, Belgium.
LeE, B., Yu, S. \& JuAn, H. (2004). The model of surface roughness inspection by vision system in turning. Mechatronics 14(1), 129-141.

Maes, F., Collignon, A., Vandermeulen, D., Marchal, G. \& Suetens, P. (1997). Multimodality image registration by maximization of mutual information. IEEE Trans Med Imaging 16(2), 187-198.

RAADNU, S. (2005). Wear particle analysis-utilization of quantitative computer image analysis: a review. Tribol Int 38(10), 871-878.

Rymuza, Z. (2007). Tribology of polymers. Arch Civ Mech Eng 7(4), $177-184$.

Soleimani, S. (2014). Computer vision system for wear analysis. $\mathrm{PhD}$ thesis, Ghent University, Ghent, Belgium.

Soleimani, S., Rooms, F. \& Philips, W. (2013). Efficient blur estimation using multi-scale quadrature filters. Signal Processing 93(7), 1988-2002.

Soleimani, S., Sukumaran, J.P., Douterloigne, K., Rooms, F., Philips, W. \& DE BAETS, P. (2012). Correction, stitching and blur estimation of micro-graphs obtained at high speed. In Advanced Concepts for Intelligent Vision Systems, Blanc-Talon, J., Philips, W., Popescu, D., Scheunders, P. \& Zemcik, P. (Eds.), pp. 84-95. Berlin Heidelberg: Springer.

SukUmaran, J. (2011). Roll-slip phenomenon of polymer composites: online analysis assisted by computer vision. In 12th FEA PhD Symposium, Faculty of Engineering and Architecture, Ghent University, Ghent, Belgium.

Sukumaran, J. (2014). Vision assisted tribolography of rollingsliding contact pf polymer-steel pairs. $\mathrm{PhD}$ thesis, Ghent University, Ghent, Belgium.

Sukumaran, J., Soleimani, S., De Baets, P., Rodriguez, V., Douterloigne, K., Philips, W. \& Ando, M. (2012). High-speed imaging for online micrographs of polymer composites in tribological investigation. Wear 296, 702-712.

Tasan, Y., De Rooij, M. \& Schipper, D. (2005). Measurement of wear on asperity level using image-processing techniques. Wear 258(1), 83-91.

Thévenaz, P. \& Unser, M. (2000). Optimization of mutual information for multi-resolution image registration. IEEE Trans Image Process 9, 2083-2099.

VAZIRI, M., SPURR, R. \& STOTT, F. (1988). An investigation of the wear of polymeric materials. Wear 122(3), 329-342.

VILLE, O. \& HeIKKILA, J. (2007). Image registration using blur-invariant phase correlation. IEEE Signal Process Lett 14(7), 449-452.

WANG, W., Wong, P. \& Zhang, Z. (2000). Experimental study of the real time change in surface roughness during running-in for pehl contacts. Wear 244, 140-146.

Zhang, J., Korsten, M. \& Regtien, P. (2003). A vision system for online wear detection. In Proceedings XVII IMEKO World Congress, pp. 1960-1964, Dubrovnik, Croatia.

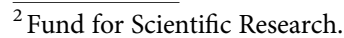

